\title{
UN METODO PER LA MISURA ASSOLUTA DELL'AC- CELERAZIONE DI GRAVITÀ: IL ROTOGRAVIMETRO (*)
}

\author{
EnRico Medi
}

Per eseguire una misura assoluta di grarita si possono, almeno in teoria, seguire diverse vie sperimentali. Una espressione matematica che contenga la $g$, permette di ricarare questo dato quando si possano determinare sperimentalmente le altre grandezze (isiche. Cosi, per esempio, la velocita finale di un corpo, che cade liberamente nel vuoto, i una funzione della gravita e dell'altezza di cadula; la forma della traiettoria percorsa da un mobile nello spazio, senza attrito con l'aria, è una funzione della gravita; le oscillazioni di un pendolo hanno un periodo legato alla $g$ da una espressione ben nota. Questo ultimo tipo di esperienze è quello più comunemente usato per la determinazione assoluta di $g$.

Sono noti gli artifici necessari affinché la misura sia corretta da errori. Per eliminare tali errori bisogna tenere conto della elasticita dei materiali, degli effelti di temperatura, delle perturbazioni dovute agli attriti ecc. Per ottenere una misura adeguatamente precisa è necesario determinare le lunghezze e i periodi in modo da ricavare almeno la quinta cifra simnificativa esente da errori, il che comporta il dover ripetere un numero sufficiente di rolte le misure. In particolare, per conoscere con la dovuta esattezza il periodo, è necessario far compiere al pendolo un grande numero di oscillazioni.

Date tali difficoltà si è pensato di ricorrere ad un artificio sperimentale diverso da quelli comunemente usati, atto a fornire il valore della gravità partendo dalla conoscenza di un periodo e di una distanza. determinabili con maggiore facilitì e precisione ed evitando molte cause di errore, soprattutto quelle provocate dagli effetti della temperatura.

Il metodo consiste nel far ruotare un liquido intorno ad un asse parallelo alla direzione della qravita. Se il liquido è perfetto, la sua superficie libera diventa una superficie equipotenziale: la risultante della accelerazione centrifuga e della gravità è in ogni punto della

I* Il metodo è stato esposto dall'A. all'Association Internationale de Geodésie nella $X$ Assemblea Generale dell U.G.G.I. - Roma, 1954. 
superficie, normale alla superficie stessa. Essendo l'accelerazione centrifuga proporzionale alla distanza dall'asse, la superficie è un paraboloide di rivoluzione avente come asse di simmetria l'asse di rotazione. L'equazione del paraboloide è la seguente, essendo il polo all'origine degli assi,

$$
y=\frac{(1)^{2} x^{2}}{2 g}
$$

Come si vede chiaramente essa é funzione di un rapporto fra accelerazioni e non fra le forze, quindi gli effetti della temperatura sono nulli. Qualunque sia la densità del liquido, purche perfetto, la lorma del paraboloide e le sue caratteristiche sono identiche.

Con semplici passaggi si trova la distanza locale del paraboloide

$$
\operatorname{per}\left\{\begin{array}{l}
y=f \\
x=2 f
\end{array} \text { di cui } T=2 \pi \sqrt{\frac{2 f}{g}}\right.
$$

e quindi il valore della accelerazione di gravità

$$
g=8 \pi^{2} n^{2} f \quad n=\frac{I}{T}
$$

L'espressione ricorda quella del pendolo, pur non avendo nulla in comme dal punto di vista della impostazione del metodo.

Per avere $g$, es sufficiente determinare il periorlo di rotazione e la distanza focale.

La prima misura é facilitata dal fatto di essere in presenza di un moto rotatorio a velocità angolare costante.

Ciò permette di usare un sistema a moltiplicazione di freyuenza. e di confrontare la frequenza cosi ottenuta con un oscillatore campione.

Per la determinazione della frequenza di rotazione si possono usare diversi metodi: la scelta del metodo più adatto è suggerita dai risultati delle esperienze in corso. È necessario innanzi tutto disporre di un misuratore di tempi, atto a dare la precisione al decimillesimo di secondo. Lo strumento adatto a ciò é l’orologio a quarzo, o un oscillatore tarato su quello. Nella rotazione il disco sostenente il liquido. mediante un sistema di accoppiamenti a rulli a frizione. fa ruotaro un asse con una frequenza avente l'ordine di grandezza di alcune centinaia di rotazioni al secondo.

Il rapporto fra la frequenza di rotazione del disco e quella del-

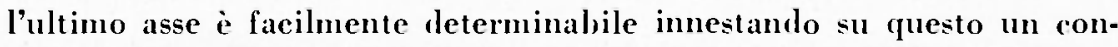
tagiri. L'ultimo asse porta una bobina di dieci spire sfasate fra loro 
di trentasei gradi; ruotando si genera in un indotto fisso una corrente sinusoidale avente una frequenza di alcume mirliaia di hertz. Questa modulazione è amplificata e confrontata con la frequenza campione mediante sistemi ben noti.

Un altro artificio consiste nell'eseguire fotografie del conta griri e di un cronografo campione, mediante illuminazioni di Jrevissima durata.

Appare ben chiaro come con alcume centinaia di rotazioni è possibile ottenere una precisione più che soddisfacente nella determinazionc del periodo. Bastano alcune diecine di minuti, invece delle molte ore di sperimentazione necessarie col sistema pendolare, per fare la misura.

Per la determinazione della distanza focale si può usare uno dei vari metodi suggeriti dallottica per la misura delle distanze focali dei sistemi ottici. Si ricordi che, nel caso del paraboloide di rotazione, per il fuoco non esiste aberrazione di sfericita e astigmatismo. Il fuoco è rigorosamente stigmatico per un punto all'infinito. Tutti i raggi paralleli all'asse, dopo la riflessione, passano per il fuoco e viceversa.

La individuazione della posizione del fuoco può essere eseguita con il segruente dispositivo. Una sorgente luminosa puntiforme posta nel fuoco di origrine dopo la riflessione nella superficie del paraboloide ad un fascio di ragcri paralleli che incontrano la superficie di uno specchio piano posto normalmente allasse.

I rargri riflessi da questo specchio sono ancora paralleli e damo luogo, dopo lutima riflessione sulla superficie del paraboloide, ad una immagrine reale, che coincide esattamente con la sorgente.

Un microscopio, dotato di minima profondità di fuoco, assicura la coincidenza della sorgente con la sua immagrine reale: quando si realizza questa condizione, è individuata la posizione del fuoco e si è certi che lo specchio piano i normale allasse. Stabilita la posizione del fuoco, si determina, con il medesimo microscopio, la posizione del vertice della parabola, unico punto fisso nella rotazione.

La distanza fra le due posizioni è la distanza focale cercata. Per ottenere una misura ancora più precisa e indipendente da campioni di misura, si fa funzionare il paraboloide ruotante come uno specchio di un interferometro di Michelson e quindi si può determinare, in lunghezze donda di una radiazione nota, la distanza focale, come differenza fra due cammini ottici. Compensando tale differenza si riottengono le frange di ordine zero o centrali. 
Quanto è qui esposto parte dall'ipotesi che si tratti di un liquido perfetto, nel quale cioè siano da trascurare le forze di interazione molecolare. In realtà ogni liquido ha una certa tensione superficiale che perturba la forma della superficie del paraboloide. Si usano perciò nella esperienza liquidi di bassissima tensione di vapore e conseguentemente di piccola tensione superficiale. Realizzando queste condizioni la perturbazione è trascurabile. In ogni modo, in una nota immediatamente seguente questa, è publjlicato il calcolo rigoroso della perturbazione. Un risultato fondanentale del calcolo è che bastano due determinazioni sperimentali per conoscere la perturbazione e quindi correggere l'errore da essa apportato. Date le sue caratteristiche al dispositivo è dato il nome di "rotogravimetro ". In un prossimo lavoro saramno pubblicati i particolari sperimentali e i primi risultati delle misture.

\section{Roma - Istituto Nazionale di Geofisica - Settembre 1954}

\section{RIASSUNTO}

E esposto un metodo per la misura assoluta della gravita, basato sulla forma assunta dalla superficie libera di un liquido posto in rotazione, attorno ad un asse parallelo alla direzione dell accelerazione di gravità nel laogo di osservazione.

Misurando la distanza focale del paraboloide di rivoluzione che cosi si ottiene, e il periodo di rotazione del sistema, ¿ possibile ricavare il valore di g. Il metodo fa si che gli effetti dovuti alla temperatura non influiscano sulla misura e che siano eliminate altre cause di errore, presenti in altri metodi.

\section{$S U M M A R Y$}

A method is discussed jor an absolute measurement of the constant of gravity. This method is based on the form tatien by a liquid set in rotation around an axis parallel to the direction of the accoleration of gravity at the place of observation.

By measuring the foca! distance of the revolution paraboloid thus obtained, and the period of rotation of the system, it is possible to obtain a value for g. There are no temperature effects with this method measurement, and further sources of errors, normally present in other methods, are eliminated. 